\title{
Estudio de la inactivación de microorganismos deteriorativos en jugos de manzana y melón tratados por luz pulsada y ultrasonido
}

\section{Study of the inactivation of spoilage microorganisms in apple and melon juices by pulsed light and ultrasound}

\author{
Ferrario, Mariana (1), Guerrero, Sandra (1) \\ (1) Departamento de Industrias, Facultad de Ciencias Exactas y Naturales, Universidad de Buenos Aires, Argentina. \\ Contacto: sguerrero@di.fcen.uba.ar
}

RECIBIDO: 29/2/2016 - APROBADO: 13/6/2016

\begin{abstract}
Resumen
En este estudio se investigó el efecto de la luz pulsada $\left(0-71,6 \mathrm{~J} / \mathrm{cm}^{2}, \mathrm{~T}<20^{\circ} \mathrm{C}\right)$ en sistema estanco $\left(\mathrm{LP}_{\mathrm{e}}\right)$ en la inactivación de esporas de Alicyclobacillus acidoterrestris ATCC 49025, células de Saccharomyces cerevisiae KE 162 y Escherichia coli ATCC 35218 en jugos naturales de manzana (Pyrus malus L, var. Granny Smith, pH: 3,4; $11,4{ }^{\circ}$ Brix) y melón (Cucumis melo, var. rocío de miel; $\mathrm{pH}: 5,7 \pm 0,2 ; 8,4 \pm 2,5^{\circ} \mathrm{Brix}$ ). Por otra parte, se estudió el efecto de LP en flujo continuo $\left(\mathrm{LP}_{\mathrm{c}}, 0-0,73 \mathrm{~J} / \mathrm{cm}^{2}, 0-0,0175 \mathrm{~J} / \mathrm{ml}, 155 \mathrm{ml} / \mathrm{min}, \mathrm{T}: 25^{\circ} \mathrm{C}\right)$ combinado o no con ultrasonido (US, $20 \mathrm{kHz}, 80 \%, 30 \mathrm{~min}, \mathrm{~T}: 25^{\circ} \mathrm{C}$ ) en la inactivación de E. coli en jugo de manzana. El tratamiento $\mathrm{LP}_{\mathrm{e}}$ simple provocó hasta 1,9-6,2 y 1,0-2,1 reducciones logarítmicas de A. acidoterrestris, S. cerevisiae y E. coli en jugos de melón y-manzana, respectivamente. Los tratamientos de $\operatorname{LP}_{c}$ y US provocaron una reducción de 3,1 y 2,7 ciclos logarítmicos de E. coli en jugo de manzana, respectivamente. La combinación US+LP ${ }_{c}$ resultó aditiva dado que se logró una reducción de 5,7 ciclos logarítmicos. Los modelos bifásico y de Weibull brindaron mejor ajuste y estimación de los parámetros que el modelo de Coroller. El jugo de manzana resultó altamente aceptado por un grupo de consumidores que resaltaron su sabor a manzana natural.

Palabras clave: Tecnologías emergentes, tratamientos combinados, jugos frutales.
\end{abstract}

\begin{abstract}
This study was aimed to investigate the effect of pulsed light $\left(0-71.6 \mathrm{~J} / \mathrm{cm}^{2}, \mathrm{~T}<20^{\circ} \mathrm{C}\right)$ in batch mode operation $\left(\mathrm{PL}_{\mathrm{s}}\right)$ in the inactivation of Alicyclobacillus acidoterrestris ATCC 49025 spores, Saccharomyces cerevisiae KE162 and Escherichia coli ATCC 35218 cells in natural squeezed apple (Pyrus malus L, var Granny Smith, pH: 3,4; 11,4 ${ }^{\circ}$ Brix) and melon (Cucumis melo, var. Honeydew, $\mathrm{pH}: 5,7 \pm 0,2,8,4 \pm 2.5^{\circ} \mathrm{Brix}$ ) juices, and continuous flow-through pulsed light system $\left(\mathrm{PL}_{\mathrm{c}}, 0-0,73 \mathrm{~J} / \mathrm{cm}^{2}, 0-0,73 \mathrm{~J} / \mathrm{cm}^{2}, 0-0,0175 \mathrm{~J} / \mathrm{ml}, 155 \mathrm{ml} / \mathrm{min}, \mathrm{T}<25^{\circ} \mathrm{C}\right)$ single or combined with ultrasound (US, $30 \mathrm{~min}, \mathrm{~T}: 25^{\circ} \mathrm{C}$ ) on the inactivation of E. coli in apple juice. $\mathrm{PL}_{\mathrm{s}}$ led up to $1,9-6,2 \log$ reductions in melon juice; and 1,0-2,1 in apple juice for A. acidoterrestris, S. cerevisiae and E. coli, respectively. Single $\mathrm{PL}_{c}$ reduced E. coli population by $3,1 \log$ cycles, while single US reduced $2,7 \log$ cycles. The combination $\mathrm{US}_{+} \mathrm{PL}_{\mathrm{c}} \mathrm{re-}$ sulted additive as a reduction of 5,7 log cycles was achieved. Biphasic and Weibull models compared to the Coroller model allowed better fit and more accurate estimation of parameters. Processed apple juice was well accepted by a group of consumers who highlighted its fresh natural apple tasting.

Keywords: Emerging technologies, hurdle technology, fruit juices.
\end{abstract}

\section{Introducción}

Los consumidores optan cada vez más por el consumo de alimentos sometidos a tratamientos menos severos, más saludables, con bajos niveles de sal, azúcares, grasas, ácidos, y con la sustitución total o parcial de conservantes químicos. El procesamiento térmico, si bien asegura la seguridad y la vida útil de los jugos frutales, provoca un detrimento en la calidad nutricional y sensorial de los productos.

Los jugos frutales, debido a su alta acidez, son deteriorados por levaduras, principalmente Saccharomyces spp.(Martínez, et al., 2000), hongos como Aspergillus ochraceus, y bacterias ácido-lácticas como Lactobacillus y Leuconostoc spp.(Stratford, et al., 2000). Además, Alicyclobacillus acidoterrestris es una bacteria termoacidofílica, resistente al calor y capaz de sobrevivir a los procedimientos de pasteurización normalmente aplicados a los jugos frutales (Bahçeci, et al., 2005). Por lo tanto, las endoesporas pueden germinar y producir compuestos de mal sabor (Smit, et al., 2010).

En las últimas décadas se ha profundizado en la utilización de tecnologías emergentes de preservación aplicadas de modo individual o en una estrategia combinada para garantizar la 
seguridad y retener o mejorar la calidad de los alimentos (Alzamora, et al., 2011). Este tipo de procesamiento reduce la carga microbiana y, al mismo tiempo, permite mejor retención de flavor, textura, color y contenido de nutrientes en relación a los tratamientos térmicos convencionales.

La implementación de luz pulsada (LP) en la inactivación de microorganismos ha ganado interés debido al corto tiempo de procesamiento involucrado (Krishnamurthy, et al., 2010) y su alta efectividad en la inactivación tanto de células vegetativas como esporas en una amplia variedad de alimentos como leche, harina de maíz, pimientos, manzana fresca cortada y carne, entre otros (Jun, et al., 2003; Gómez-López, et al., 2007; Krishnamurthy, et al., 2008; Gómez, et al., 2012; Hierro, et al., 2011). La exposición a LP promueve la formación de dímeros de pirimidina que impiden el proceso de replicación (efecto fotoquímico) (Gómez-López, et al., 2007). Asimismo, Wekhof (2000) y Ferrario et al. (2013a) han reportado disrupción de la membrana como resultado de un sobrecalentamiento momentáneo (efecto fototérmico).También se han reportado daños en la estructura de las células microbianas como encogimiento de la membrana (efecto fotofísico) (Krishnamurthy, et al., 2010). Es posible que estos mecanismos coexistan y que la importancia relativa de cada uno dependa de la fluencia, es decir, la potencia de radiación total incidente, y del microorganismo blanco (Gómez-López, et al., 2007).

Por otra parte, se ha propuesto la utilización de ultrasonido de alta intensidad (US) en la preservación de alimentos. El efecto letal de US se atribuye al fenómeno de cavitación en el que las burbujas implosionan generando altas temperaturas y presiones en el punto de colisión (Knorr, et al., 2004; Guerrero, et al., 2001 y 2005). El mecanismo de inactivación se debe principalmente al adelgazamiento de la pared, el calentamiento localizado y la producción de radicales libres por la sonólisis del agua (Piyasena, et al., 2003). La aplicación de US como único factor de preservación resulta inefectiva en la inactivación de esporas bacterianas y también requiere de largos tiempos de procesamiento para la inactivación de microorganismos. Sin embargo, se ha demostrado que la aplicación de US en una estrategia combinada con otras tecnologías tradicionales o emergentes resultó altamente efectiva para la inactivación de microorganismos patogénicos o deteriorativos (Guerrero, et al., 2001 y 2005; Ferrante, et al., 2007).

El objetivo de este trabajo consistió en: i) Evaluar y caracterizar el efecto individual de $\mathrm{LP}$ en sistema estanco $\left(\mathrm{LP}_{\mathrm{e}}\right)$ en la inactivación de esporas de Alicyclobacillus acidoterrestris ATCC 49025, y células de Saccharomyces cerevisiae KE 162 y Escherichia coli ATCC 35218 en jugos naturales de manzana y melón; ii) evaluar y caracterizar el efecto de LP en flujo continuo $\left(\mathrm{LP}_{\mathrm{c}}\right)$ combinado o no con US (US+LP $)$ en la inactivación de Escherichia coli en jugo de manzana; iii) analizar la aceptabilidad global del jugo de manzana tratado con US+LP

\section{Materiales y Métodos}

\section{Obtención de esporas y preparación de los inóculos}

Los ensayos se llevaron a cabo utilizando esporas de Alicyclobacillus acidoterrestris ATCC 49025 y células de Saccharomyces cerevisiae KE 162 y Escherichia coli ATCC 35218. Se preparó el inóculo inicial transfiriendo dos asadas de un cultivo fresco en estría a un erlenmeyer que contenía 20 ml de caldo Tripteína Soya (Biokar Diagnostics, Beauvais, Francia) para E. coli o caldo Sabouraud (Biokar Diagnostics, Beauvais, Francia) para levaduras. Los caldos se incubaron a $37^{\circ} \mathrm{C}$ durante $18 \mathrm{~h} \mathrm{o} 27^{\circ} \mathrm{C}$ durante $24 \mathrm{~h}$, respectivamente. Los inóculos se centrifugaron $5 \mathrm{~min}$ a $5000 \mathrm{rpm}$ (Labnet, EE.UU) y fueron resuspendidos en agua peptonada $0,1 \% \mathrm{p} / \mathrm{v}$.

En el caso de las esporas de $A$. acidoterrestris, el inóculo se obtuvo transfiriendo una asada de cultivo fresco a un erlenmeyer que contenía $20 \mathrm{ml}$ caldo BAM y posterior incubación a $43{ }^{\circ} \mathrm{C}$ por $24 \mathrm{~h}$. La producción de esporas se llevó a cabo sembrando el inóculo en botellas con medio A. acidoterrestris e incubándolo una semana a $43 \pm 1{ }^{\circ} \mathrm{C}$. Las esporas fueron removidas según el protocolo de Silva y Gibbs (2001) y mantenidas a $-18 \pm 1{ }^{\circ} \mathrm{C}$ hasta su utilización.

\section{Materia prima}

Se utilizaron jugos naturales exprimidos de manzana (Pyrus malus $L$, var, Granny Smith; $\mathrm{pH}: 3,4 \pm 0,2 ; 12,6 \pm 0,1^{\circ} \mathrm{Brix}$; $45,7 \pm 2,0 \mathrm{NTU}$; tamaño partícula: $712 \mathrm{~nm}$ ) y melón (Cucumis melo, var. rocío de miel; $\mathrm{pH}: 5,9 \pm 0,3 ; 10,6 \pm 0,3{ }^{\circ} \mathrm{Brix}$; $166,5 \pm 7,0 \mathrm{NTU}$; tamaño partícula: $478630 \mathrm{~nm}$ ). Las frutas adquiridas en el mercado local se sanitizaron y se exprimieron en el laboratorio en condiciones asépticas con un extractor de jugos de tipo hogareño (Bluesky, China). El jugo se filtró utilizando gasa estéril y se centrifugó durante 10 minutos a 1000 o 5000 rpm (Eppendorf, modelo 5804 R, Alemania) con el fin de disminuir la cantidad de pulpa. Todos los jugos fueron envasados al abrigo de la luz y almacenados a $-18^{\circ} \mathrm{C}$ hasta su posterior utilización.

\section{Tratamientos de luz pulsada en modo estanco $\left(L P_{e}\right)$ y continuo $\left(L P_{c}\right)$}

Los tratamientos por luz pulsada (LP) se llevaron a cabo en un sistema RS-3000B Steripulse-XL (Xenon Corporation, Woburn, MA, EE.UU), que produce una radiación policromática en un rango de 200 a $1100 \mathrm{~nm}$. El sistema consistió de un módulo controlador RC-747, una cámara de tratamiento que aloja una lámpara de xenón y un sistema de refrigeración anexado a dicha lámpara que evita el sobrecalentamiento durante la operación. El sistema generó pulsos de luz de alta intensidad con una velocidad de 3 pulsos de $360 \mu$ se duración por segundo. Cada pulso entregó $1,27 \mathrm{~J} / \mathrm{cm}^{2}$ con una entrada de $3800 \mathrm{~V}$ a $1,9 \mathrm{~cm}$ de la lámpara.

Se añadieron $100 \mu \mathrm{L}$ de inóculo a $4,9 \mathrm{ml}$ de jugo dentro de una placa de Petri, alineada respecto a la lámpara y a una distancia de $10 \mathrm{~cm}$ de la misma, sumergida en hielo en escamas para evitar el aumento de temperatura. Se irradió entre 0 y $60 \mathrm{~s}\left(0\right.$ a $\left.71,64 \mathrm{~J} / \mathrm{cm}^{2}\right)$.

Los tratamientos $\mathrm{LP}_{c}$ se realizaron en el equipo descripto para $\mathrm{LP}_{\mathrm{e}}$ pero con algunas modificaciones. En cada experiencia se recircularon $250 \mathrm{ml}$ de jugo a $155 \mathrm{ml} / \mathrm{min}$, empleando una bomba peristáltica (CPX-400, Cole Parmer, Illinois, EE.UU.). El sistema diseñado para tal fin consistió en dos tubos de cuarzo transparentes a la luz (d.i. 0,001 m) localizados a 0,1 m de la lámpara (ubicados en la zona donde la dosis emitida por la lámpara es constante) y conectados en serie por mangueras flexibles autoclavables (Cole-Parmer, Masterflex, L/S 15, Illinois, EE.UU.) que descargaban a un vaso de $400 \mathrm{ml}$ de capacidad. El vaso se colocó en un baño de hielo, mientras que los dos tubos de cuarzo (cada tubo con una longitud total de exposición a la LP de 0,20 m y cubiertos 
con papel aluminio en las zonas de no exposición) estaban insertos en un sistema de enfriamiento constituido por una unidad de aluminio sellada por la cual circulaba un refrigerante (etilenglicol, calidad industrial) para evitar incrementos en la temperatura del sistema debido al tratamiento con LP. Para cada tratamiento, $250 \mathrm{ml}$ de jugo inoculado y sometido o no al tratamiento ultrasónico, fue expuesto al tratamiento por $\mathrm{LP}_{\mathrm{c}}\left(10 \mathrm{~min}, 0,15 \mathrm{~J} / \mathrm{cm}^{2}\right)$. Como control, se emplearon muestras inoculadas sin tratamiento $\mathrm{LP}_{c}$.

Previo al uso del dispositivo de $\mathrm{LP}_{c}$, la unidad fue completamente enjuagada con agua destilada estéril, mientras que una vez finalizados los tratamientos se realizaba un lavado con una solución de hipoclorito de sodio al $5 \% \mathrm{v} / \mathrm{v}$ durante 10 minutos y un posterior pasaje de agua destilada.

Las diferentes dosis de LP se obtuvieron alterando el número de pulsos aplicados (Gómez, et al., 2012). Las experiencias se llevaron a cabo por triplicado para cada condición ensayada. Las medidas de fluencia se realizaron con una cabeza piroeléctrica modelo ED500 (Gentec Electro-Optics, Québec, Canadá) conectada a un osciloscopio modelo TDS 2014 (Tektronix, Beaverton, EE.UU), con una apertura de cubierta de $20,3 \mathrm{~cm}^{2}$. La temperatura se monitoreó durante la irradiación empleando una termocupla tipo $\mathrm{T}$ conectada a un registrador de datos Digi-Sense modelo 69202-30 (Barnant Company Division, Barrington, EE.UU).

\section{Tratamiento de ultrasonido de alta intensidad}

En el caso de la aplicación de US simple el tratamiento ultrasónico tuvo lugar en un recipiente de doble camisa de $600 \mathrm{ml}$ de capacidad (diámetro interno $8 \mathrm{~cm}$; altura 13 $\mathrm{cm}$ ) conectado a un baño termostático (HAAKE, modelo Rotovisco, RV12, Alemania), para alcanzar una temperatura $\left(\mathrm{T}_{\mathrm{US}}\right)$ de $25 \pm 1{ }^{\circ} \mathrm{C}$ en el vaso. Se colocaron $245 \mathrm{ml}$ de jugo de manzana en el vaso y se inició el proceso de sonicación. La temperatura del sistema aumentó debido a la cavitación, estabilizándose luego de aproximadamente $3 \mathrm{~min}$ para luego mantenerse constante a lo largo de toda la experiencia, gracias a la recirculación de agua del baño termostático. A continuación, se añadieron $5 \mathrm{ml}$ del inóculo $\left(\sim 1 \times 10^{7}\right.$ $\mathrm{UFC} / \mathrm{ml}$ ). Debido al burbujeo generado por el proceso de cavitación, los sistemas siempre se mantuvieron agitados desde el comienzo de cada experiencia. Para los estudios cinéticos, $1 \mathrm{ml}$ de muestra se tomaba a intervalos regulares de tiempo hasta los $30 \mathrm{~min}$ de tratamiento. La temperatura de las muestras se monitoreó continuamente mediante una termocupla anexada al equipo de US. Las experiencias se evaluaron por triplicado.

\section{Determinación de microorganismos sobrevivientes}

Luego de la aplicación de los tratamientos y previo a la siembra, las esporas de A. acidoterrestris fueron calentadas $10 \mathrm{~min}$ a $80 \pm 1{ }^{\circ} \mathrm{C}$, con el objetivo de estimular su germinación e inactivar los organismos vegetativos (Vercammen, et al., 2012). Las muestras irradiadas a intervalos de tiempo seleccionados fueron apropiadamente diluidas y sembradas mediante un plaqueador orbital (Autoplate 4000, Spiral Biotech, EE.UU) por duplicado en BAM e incubadas $72 \mathrm{~h} \mathrm{a} 43 \pm 1{ }^{\circ} \mathrm{C}$. En el caso de E. coli y $S$. cerevisiae se sembraron en agar Tripteína Soya o agar Papa Dextrosa (Britania, Buenos Aires, Argentina) e incubadas a $37^{\circ} \mathrm{Co} 27^{\circ} \mathrm{C}$, respectivamente, durante 24 a 48 h. Las curvas de supervivencia se generaron a partir de los datos experimentales, graficando $\log \mathrm{N} / \mathrm{N}_{\mathrm{o}}$ (donde $\mathrm{N}$ es el número de UFC/ml a un tiempo dado y $\mathrm{N}_{\mathrm{o}}$ el número inicial de UFC/ml) versus el tiempo de tratamiento.

\section{Modelado matemático}

Uno de los modelos para estudiar la cinética de inactivación de los diferentes microorganismos por LP fue el modelo de distribución de frecuencias de resistencias tipo Weibull (Peleg y Cole, 1998):

$S(t)=\log \left(\frac{N}{N_{0}}\right)=-b \cdot t^{n}$

donde $S$ es la fracción de supervivientes y $b$ y $n$ son los parámetros de escala y de forma, respectivamente. Los valores de $b$ y $n$ luego fueron usados para generar las curvas de frecuencia de resistencias utilizando la siguiente ecuación:

$\frac{d \phi}{d t_{c}}=b \cdot n \cdot t_{c}^{n-1} \exp \left(-b \cdot t_{c}^{n}\right)$

donde $t_{c}$ es una medida de la resistencia o sensibilidad del microorganismo al tratamiento aplicado y es la distribución de tipo Weibull correspondiente a $t_{\mathrm{c}}$, Asimismo, se calcularon otros parámetros estadísticos de la distribución (moda, media, varianza y coeficiente de sesgo) (Peleg y Cole, 1998).

Las curvas de inactivación fueron también ajustadas por el modelo bifásico propuesto por Cerf (1977):

$\log _{10}(N)=\log _{10}(\mathrm{No})$

$+\log _{10}\left(f * e^{-k \max _{1} * t}+(1-f) * e^{-\max _{2} * t}\right)$

donde a es la proporción logarítmica entre la fracción de la subpoblación sensible $f$ y la resistente $(1-f)$ y $k_{\max 1}$ y $k_{\max 2}$ son las tasas de inactivación de las dos subpoblaciones, respectivamente.

Una versión de cuatro parámetros del modelo propuesto por Coroller et al. (2006), basado en dos distribuciones Weibullianas mixtas de resistencias bacterianas, fue también aplicada:

$\log _{10}\left(\frac{N}{N o}\right)=\frac{1}{1+10^{\alpha}}\left(10^{-\left(\frac{t}{\delta 1}\right)^{p}+\alpha}+10^{-\left(\frac{t}{\delta 2}\right)^{p}}\right)$

donde $p$ es el parámetro de forma, $\alpha$ es la proporción logarítmica entre la fracción sensible $(f)$ y la resistente $(1-f), \delta_{1}$ y $\delta_{2}$ son los tiempos para la primera reducción decimal de la subpoblación 1 y la subpoblación 2 , respectivamente.

Todos los parámetros del modelo se calcularon aplicando el procedimiento de regresión no lineal de Infostat 2009 (InfoStat Group, FCA-UNC, Córdoba, Argentina).

La validación interna de los modelos matemáticos utilizados se realizó calculando y analizando los siguientes parámetros estadísticos: la raíz cuadrada de los cuadrados medios del error (RMSE) (Alzamora, et al., 2005a); el criterio de Akaike (AIC) (Akaike, 1973); el criterio Bayesiano Schwarz (BIC) (Quinn y Keough, 2002), y el coeficiente de determinación ajustado por los grados de libertad del modelo $\left(\mathrm{R}_{\mathrm{aj}}^{2}\right)$.

\section{Análisis sensorial}

Se realizó un test de campo con consumidores con el objetivo de evaluar la impresión global respecto de algunas caracte- 
rísticas sensoriales del jugo de manzana exprimido. En la prueba de impresión global se empleó una escala hedónica de 9 puntos balanceada, con extremos que fueron desde "me disgusta extremadamente" a "me gusta extremadamente". Se realizaron dos preguntas abiertas direccionadas acerca del gusto o disgusto del producto para su respuesta en una determinada secuencia, según el puntaje otorgado en la prueba de impresión global. Luego, en el mismo formulario, se incluyó la evaluación de atributos particulares en escalas cortas de aceptabilidad, punto justo y/o intensidad (sabor a manzana y dulzor), mediante escalas cortas de intensidad, con extremos de "sin sabor a manzana" a "sabor a manzana muy intenso" y de "no dulce" a "muy dulce". El sabor ácido se evaluó por medio de una escala de punto justo de cinco puntos con el fin de determinar si este atributo se encontraba en adecuada intensidad. Se realizó un análisis de conglomerados (método de agrupamiento, promedio ponderado y distancia Euclidea) para vislumbrar la existencia de segmentación en la preferencia de los consumidores por el jugo de manzana procesado mediante $\mathrm{US}+\mathrm{LP}_{\mathrm{c}}$. Se aplicó un Análisis de Componentes Principales (PCA) para ilustrar la relación entre los puntajes obtenidos en el test de impresión global con los correspondientes a la evaluación de los atributos del jugo. Se consideró un adecuado ajuste del análisis de conglomerados y del PCA con un valor de coeficiente CCC cercano a 1 (Lawless, 2013).

\section{Resultados}

\section{Efectividad del tratamiento $\mathrm{LP}_{\mathrm{e}}$}

La Figura 1 muestra el comportamiento de células de $S$. cerevisiae y E. coli y esporas de A. acidoterrestris en jugos de manzana y melón procesados mediante $\mathrm{LP}_{\mathrm{e}}$. En general, las curvas de inactivación presentaron una importante concavidad hacia arriba con un marcado descenso durante los primeros $10 \mathrm{~s}$ de procesamiento. En ningún caso se observó presencia de un hombro inicial. Solo la curva de inactivación correspondiente a $S$. cerevisiae en jugo de manzana presentó forma sigmoidea. La exposición a $60 \mathrm{~s}$ $\mathrm{LP}_{\mathrm{e}}$ provocó 1,9, 5,2 y 6,2 reducciones logarítmicas de esporas de A. acidoterrestris, y células de S. cerevisiae y E. coli, respectivamente, en jugo de melón. En jugo de manzana, la efectividad del tratamiento fue menor dado que se obtuvo una reducción de $\sim 1,0$ ciclo log de las esporas y las levaduras y 2,1 ciclos log para E. coli. Con lo cual, este último microorganismo resultó ser el más sensible al tratamiento.

Los datos de las curvas de supervivencia fueron modelados aplicando los modelos de Weibull (Peleg y Cole, 1998), bifásico y de Coroller (Figura 1), cuyos parámetros se ilustran en la Tabla 1. Los modelos de Weibull y bifásico ajustaron adecuadamente los datos experimentales con valores del coeficiente de determinación, $\mathrm{R}_{\mathrm{aj}}^{2}$, en el rango de $89-99 \%$ y $84-98 \%$, respectivamente. Por otra parte, el modelo de Coroller ( $\mathrm{R}_{\text {aj }}^{2} 88-99 \%$ ) solo caracterizó adecuadamente las curvas de inactivación que presentaron forma sigmoidea.

Los valores de los parámetros b y $\mathrm{n}$ fueron utilizados para obtener las distribuciones de frecuencia de resistencias correspondientes a los distintos microorganismos estudiados (Figura 2). Todas las distribuciones carecieron de moda y resultaron sesgadas a la derecha, es decir, la mayor parte de

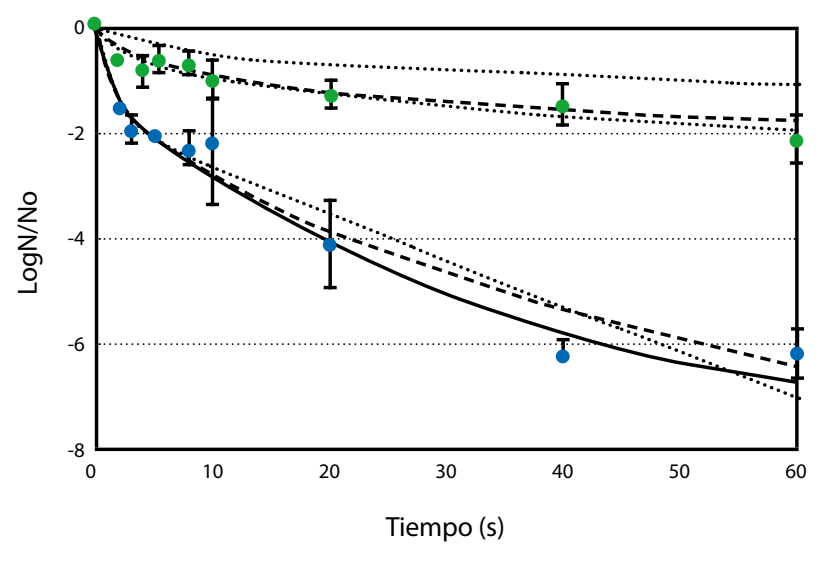

b.

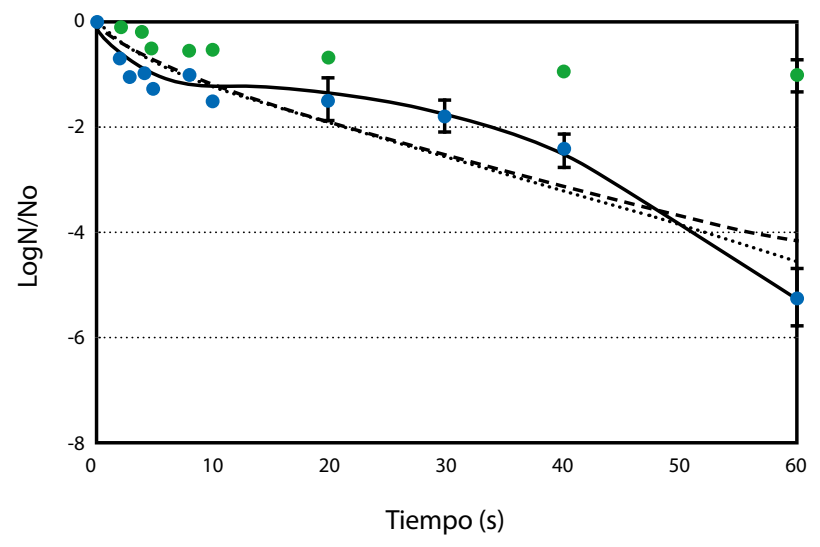

c.

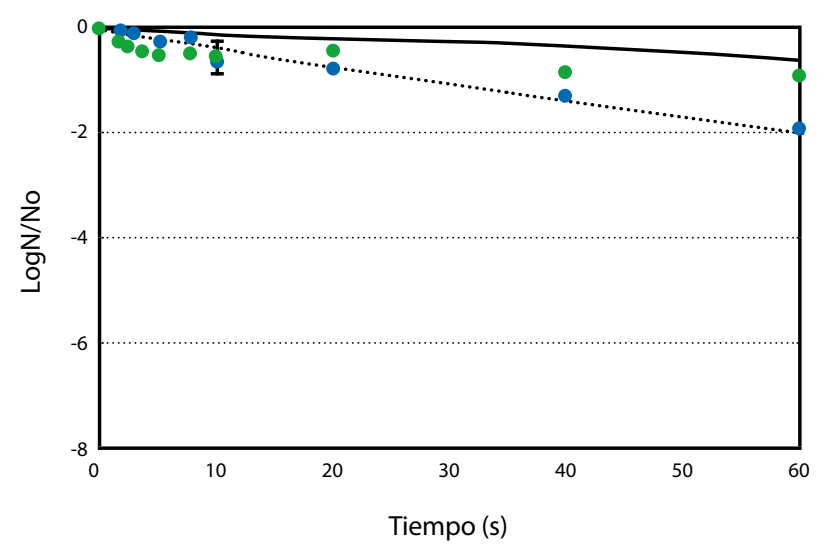

Figura 1. Curvas de supervivencia y ajuste por el modelo de Weibull (- -), modelo bifásico (…..) y modelo de Coroller (-) de células de E. coli (a), S. cerevisiae (b) y esporas de A. acidoterrestris (c) inoculados en jugos de manzana $(\bullet)$ y melón $(\bullet)$ y tratados con $\mathrm{LP}_{\mathrm{e}}$. Valor experimental (punto); predicción (línea). Desvío estándar (I)

los microorganismos en la población fueron sensibles a los efectos de la $\mathrm{LP}_{\mathrm{e}}$ a dosis muy bajas. El jugo de melón presentó menor valor de media y varianza respecto al jugo de manzana. 


\begin{tabular}{|c|c|c|c|c|c|c|c|c|c|c|}
\hline \multirow{2}{*}{\multicolumn{2}{|c|}{ Jugo / Microorganismo }} & \multicolumn{2}{|c|}{ Weibull } & \multicolumn{3}{|c|}{ Bifásico } & \multicolumn{4}{|c|}{ Coroller } \\
\hline & & \multirow{2}{*}{$\begin{array}{c}\begin{array}{c}\boldsymbol{b} \\
\left(\mathrm{s}^{-\mathrm{n}}\right)\end{array} \\
0,44^{* * *} \\
(0,05)\end{array}$} & \multirow{2}{*}{$\begin{array}{c}\begin{array}{c}\boldsymbol{n} \\
(-)\end{array} \\
0,37^{* * *} \\
(0,04)\end{array}$} & \multirow{2}{*}{$\begin{array}{c}\begin{array}{c}f \\
(-)\end{array} \\
\begin{array}{c}0,798^{* * *} \\
(0,03)\end{array}\end{array}$} & \multirow{2}{*}{$\begin{array}{c}\begin{array}{c}\boldsymbol{k m a x}_{1} \\
(1 / \mathrm{s})\end{array} \\
\begin{array}{c}1,5^{*} \\
(0,72)\end{array}\end{array}$} & \multirow{2}{*}{$\begin{array}{c}\begin{array}{c}\operatorname{kmax}_{2} \\
(1 / \mathrm{s})\end{array} \\
\begin{array}{c}0,05^{* *} \\
(0,00)\end{array}\end{array}$} & \multirow{2}{*}{ 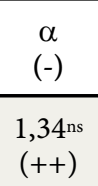 } & \multirow{2}{*}{$\begin{array}{c}\begin{array}{c}\delta_{1} \\
(s)\end{array} \\
\begin{array}{c}9,55^{\mathrm{ns}} \\
(++)\end{array}\end{array}$} & \multirow{2}{*}{$\begin{array}{c}p \\
(-)\end{array}$} & \multirow{2}{*}{$\begin{array}{c}\begin{array}{c}\delta_{2} \\
(\mathrm{~s})\end{array} \\
\begin{array}{c}9,55^{\mathrm{ns}} \\
(++)\end{array}\end{array}$} \\
\hline & E. coli & & & & & & & & & \\
\hline & S. cerevisiae & $\begin{array}{l}0,17^{* * *} \\
(0,04)\end{array}$ & $\begin{array}{l}0,45^{* * *} \\
(0,06)\end{array}$ & $\begin{array}{c}0,765^{* * *} \\
(0,07)\end{array}$ & $\begin{array}{l}0,24^{* * *} \\
(0,06)\end{array}$ & $\begin{array}{l}0,02^{* *} \\
(0,01)\end{array}$ & $\begin{array}{l}0,48^{* * *} \\
(0,09)\end{array}$ & $\begin{array}{l}6,85^{* * *} \\
(1,27)\end{array}$ & $\begin{array}{l}1,51^{* * *} \\
(0,41)\end{array}$ & $\begin{array}{l}100,4^{* * *} \\
(22,04)\end{array}$ \\
\hline \multirow{3}{*}{ Melón } & E. coli & $\begin{array}{l}0,98^{* * *} \\
(0,16)\end{array}$ & $\begin{array}{l}0,47^{* * *} \\
(0,05)\end{array}$ & $\begin{array}{c}0,985^{* * *} \\
(0,00)\end{array}$ & $\begin{array}{l}1,99 \text { ns } \\
(0,24)\end{array}$ & $\begin{array}{l}0,19^{* * *} \\
(0,05)\end{array}$ & $\begin{array}{c}6,46 \text { ns } \\
(++)\end{array}$ & $\begin{array}{l}1,24^{* *} \\
(0,45)\end{array}$ & $\begin{array}{l}0,51^{* * *} \\
(0,09)\end{array}$ & $\begin{array}{c}193,89^{\mathrm{ns}} \\
(++)\end{array}$ \\
\hline & S. cerevisiae & $\begin{array}{c}0,23^{*} \\
(0,12)\end{array}$ & $\begin{array}{l}0,71^{* * *} \\
(0,14)\end{array}$ & $\begin{array}{c}0,749^{* * *} \\
(0,15)\end{array}$ & $\begin{array}{l}2,92 \text { ns } \\
(57,1)\end{array}$ & $\begin{array}{l}0,15^{* * *} \\
(0,14)\end{array}$ & $\begin{array}{l}1,14^{* * *} \\
(0,08)\end{array}$ & $\begin{array}{l}2,17^{* * *} \\
(0,23)\end{array}$ & $\begin{array}{l}2,68^{* * *} \\
(0,27)\end{array}$ & $\begin{array}{c}35,60^{* * *} \\
(1,91)\end{array}$ \\
\hline & A. acidoterrestris & $\begin{array}{l}0,06^{* *} \\
(0,02)\end{array}$ & $\begin{array}{l}0,86^{* * *} \\
(0,08)\end{array}$ & $\begin{array}{l}0,85^{* *} \\
(0,21)\end{array}$ & $\begin{array}{l}0,03^{* *} \\
(0,01)\end{array}$ & $\begin{array}{c}0,19 \mathrm{~ns} \\
(0,35)\end{array}$ & $\begin{array}{l}-0,23^{*} \\
(0,30)\end{array}$ & $\begin{array}{l}11,94^{* *} \\
(4,59)\end{array}$ & $\begin{array}{l}2,64^{* *} \\
(0,57)\end{array}$ & $\begin{array}{c}82,13^{* * *} \\
(9,07)\end{array}$ \\
\hline
\end{tabular}

Tabla 1. Parámetros estimados correspondientes a los modelos de Weibull, bifásico y Coroller modificado representando las curvas de inactivación de células de E. coli y $S$. cerevisiae, y esporas de A. acidoterrestris en jugos tratados con $\mathrm{LP}_{\mathrm{e}}$ durante $60 \mathrm{~s}$. (valor) error estándar de cada parámetro, ns no significativo; ${ }^{* * *}$ significativo al 0,1\%; ${ }^{* *}$ significativo al $1 \%$; * significativo al 5\%, (++) error estándar mayor a 50.

a.

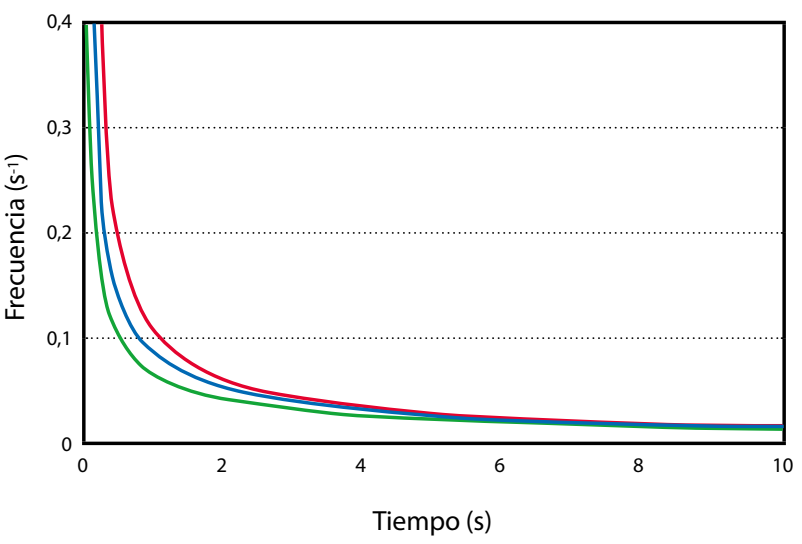

b.

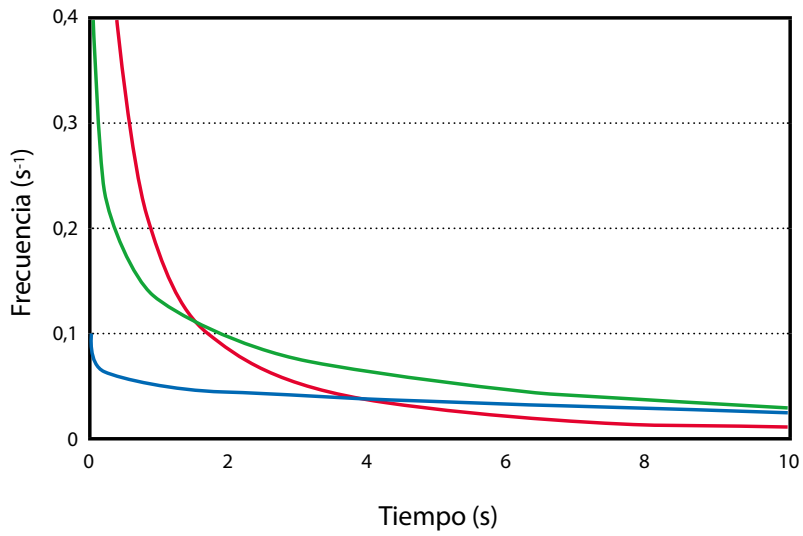

Figura 2. Distribuciones de frecuencia de resistencias correspondientes a las curvas de supervivencia de E. coli (-), S. cerevisiae (-) y esporas de A. acidoterrestris (-) en jugos naturales de manzana (a), y melón (b) tratadas con $\mathrm{LP}_{\mathrm{e}}$

\section{Efectividad de los tratamientos $L P_{c}$ y US+LP $P_{c}$ en jugo de manzana}

La curva de inactivación correspondiente a E. coli en jugo de manzana tratada con $\mathrm{LP}_{\mathrm{c}}$ resultó lineal, alcanzando hacia el final del tratamiento 3,1 reducciones logarítmicas (Figura 3a). La aplicación de 30 min de US simple provocó una reducción de 2,7 ciclos logarítmicos de E. coli y de jugo de manzana, mientras que el tratamiento combinado $\mathrm{US}+\mathrm{LP}_{c}$, provocó una reducción de 5,7 ciclos logarítmicos (Figura 3a). Por ende, la aplicación de US+LP $\mathrm{L}_{\mathrm{c}}$ mostró efecto aditivo, dado que la inactivación obtenida luego de la combinación de las tecnologías resultó ser la suma de los efectos aplicados de manera separada. La Figura $3 b$ ilustra las distribuciones de frecuencia de resistencias correspondientes a $E$. coli inoculado en jugo de manzana y tratado por $\mathrm{LP}_{\mathrm{c}}$ y US+LP $\mathrm{L}_{\mathrm{c}}$. En ambos casos las distribuciones resultaron sesgadas a la derecha, con valores similares de media y varianza. En el caso del tratamiento combinado se halló presencia de moda.

\section{Análisis sensorial}

La aceptabilidad global de las muestras de jugo de manzana procesadas por $\mathrm{US}_{+} \mathrm{LP}_{\mathrm{c}}$ presentaron un valor promedio de 6,1 en la escala hedónica de 9 puntos, correspondiente a la categoría de "me gusta algo". Si bien el jugo de manzana procesado mediante US+LP $\mathrm{L}_{\mathrm{c}}$ recibió un bajo puntaje en el test de impresión global, cuando los consumidores se segmentaron de acuerdo a sus preferencias, se encontraron dos grupos: cluster 1 (C1), conformado por 22 consumidores, englobando las categorías 2 a 5 en la escala hedónica de 9 puntos, y el cluster 2 (C2), de 55 consumidores, incluyendo las categorías 6 a 9 (no se muestran los datos). El CCC obtenido resultó 0,84 , lo cual indica un buen ajuste alcanzado con este análisis, según el criterio adoptado por Everitt et al. (2001). El cluster 2 mostró un fuerte interés en el producto, dado que la media de la impresión global para el producto fue de 7,0 puntos, correspondiente a la categoría "me gusta 
a.

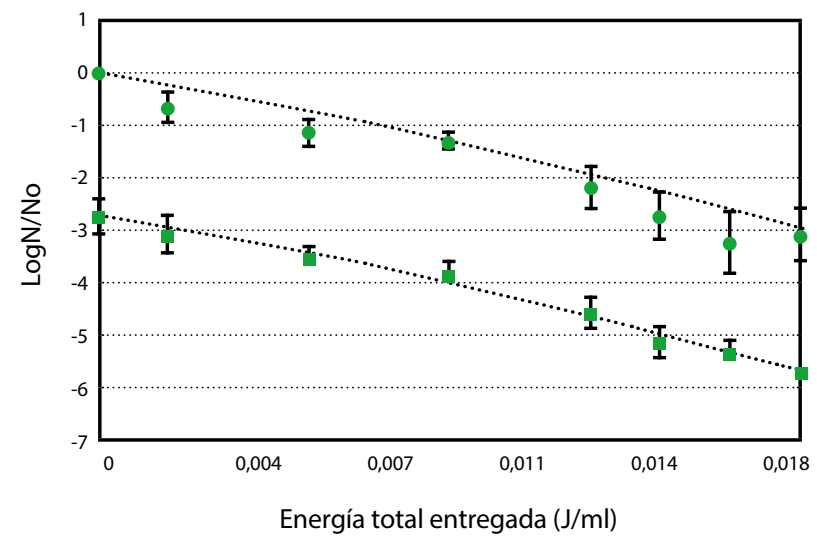

b.

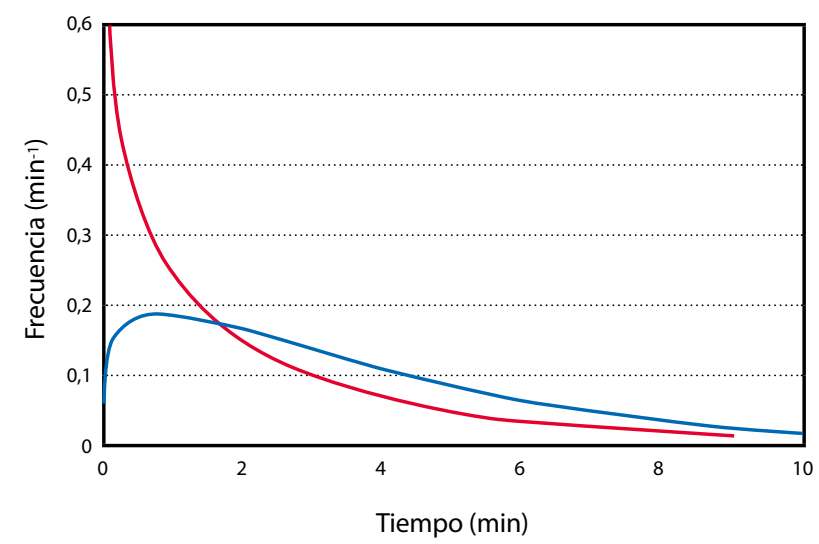

Figura 3. (a) Curvas de supervivencia y ajuste por el modelo de Weibull (......) de E. coli en jugo de manzana y tratado con

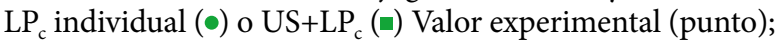
predicción (línea). Desvío estándar (I). (b) Distribuciones de frecuencia de resistencias correspondientes a E. coli en jugo de manzana procesadas por $\mathrm{LP}_{\mathrm{c}}(-)$ y $\mathrm{US}+\mathrm{LP}_{\mathrm{c}}(-)$.

moderadamente". Adicionalmente, se llevó a cabo un PCA para encontrar las relaciones entre los puntajes asignados en la escala hedónica y el porcentaje de los puntajes asignados en la escala de intensidad de sabor a manzana y de la acidez óptima del jugo de manzana natural procesado por $\mathrm{US}_{\mathrm{L}} \mathrm{LP}_{\mathrm{c}}$ (Figura 4). El CCC obtenido fue 1,0, el cual indica que se alcanzó una reducción adecuada con el análisis. Se retuvieron los dos primeros componentes principales $\left(\mathrm{CP}_{1}\right.$ y $\left.\mathrm{CP}_{2}\right)$ que

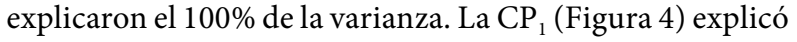
el 79,5\% y se encontró asociada positivamente con la intensidad del sabor a manzana y negativamente con la acidez óptima; mientras que la $\mathrm{CP}_{2}$ se asoció de manera positiva con ambas variables y explicó el 20,5\% de la varianza. El análisis mostró que los bajos puntajes de la escala hedónica ( 2 a 5 puntos) estuvieron asociados a valores de acidez por encima de su punto justo y bajos valores de intensidad de sabor a manzana. Por otra parte, los altos valores asignados por los panelistas en la escala hedónica ( 6 a 9 puntos) estuvieron asociados con alta intensidad y valores de acidez más cercanos al óptimo. El C2 percibió significativamente la acidez más cerca del punto justo $(\mathrm{p}<0,0001)$, exhibiendo una media de acidez de 3,6, mientras que el C1 mostró un promedio de 4,5 en la escala de punto justo.

\section{Discusión}

Las curvas de inactivación de E. coli, S. cerevisiae y esporas de $A$. acidoterrestris inoculados en jugos de manzana y melón y tratados por LP resultaron no lineales, en general con concavidad hacia arriba, a excepción de $S$. cerevisiae en jugo de manzana que resultó sigmoidea. En concordancia, numerosos estudios revelaron que las cinéticas de inactivación de diversos microorganismos inoculados en distintas matrices y tratados por LP resultaron no lineales. Se ha reportado una amplia diversidad respecto a la forma de las curvas de inactivación; en algunos casos se ha evidenciado la presencia de cola (Pataro, et al., 2011), forma sigmoidea (Izquier y Gómez-López, 2011; Lasagabaster y Martínez, 2014; Ferrario, et al., 2013b) o bifásica (Ferrario, et al., 2013b).

En este estudio se obtuvo una inactivación para E. coli de 1,0 reducciones log luego de $10 \mathrm{~s}\left(12 \mathrm{~J} / \mathrm{cm}^{2}\right)$ de exposición a LP en jugo de manzana natural exprimido. En particular, Sauer y Moraru (2009) obtuvieron una reducción en el rango de 2,5-2,7 ciclos log de E. coli ATCC 25922 y E. O157:H7, respectivamente, inoculados en jugo comercial clarificado y sidra de manzana tratados por LP en sistema estanco (13 $\mathrm{J} / \mathrm{cm}^{2}$ ). La inactivación microbiana fue más efectiva en el jugo de melón en comparación con el jugo de manzana. Esto puede atribuirse a la menor absortividad en el rango de 200 a $300 \mathrm{~nm}$ del jugo de melón (Ferrario, et al., 2013b), dado que la presencia de partículas que absorben en la región UV (lo cual se ve reflejado en el valor de absortividad de los jugos) sería perjudicial para la eficiencia del proceso de desinfección, mientras que el tamaño de partícula y la turbidez no tuvieron un rol determinante en la efectividad del proceso. Asimismo, el pH tampoco sería un factor relevante en la efectividad del tratamiento, dado que el jugo menos ácido (jugo de melón) presentó la mayor tasa de inactivación. Estos resultados concuerdan con los reportados por Sauer y Moraru (2009), quienes observaron que el tratamiento por LP resultó más efectivo en una solución modelo de baja absorbancia en el rango UV y alto $\mathrm{pH}$ (pH: 7,4) que en jugo y sidra de manzana (pH: 4).

A su vez, la efectividad del tratamiento varió según el microorganismo, siendo E. coli el más sensible. Anderson et al. (2000) también reportaron que Escherichia coli 12079 (serotipo O157:H7) resultó la cepa más sensible a LP al analizar un grupo de bacterias Gram- positivas y negativas. Sin embargo, Gómez-López et al. (2007) no encontraron un patrón de sensibilidad a LP al estudiar una amplia variedad de microorganismos.

El modelo de Weibull ajustó adecuadamente las curvas de inactivación, especialmente las de jugo de melón correspondientes a $E$. coli y a A. acidoterrestris. Sin embargo, los altos valores de varianza obtenidos (Tabla 2), incluso en el jugo de melón, sugieren que el modelo no ofreció un óptimo ajuste y/o la heterogeneidad de la respuesta (evidenciada en la presencia de colas en la distribución) fue alta.

El modelo bifásico, si bien no caracterizó adecuadamente todas las curvas de inactivación, brindó el mejor ajuste y resultó el más parsimonioso al obtener los menores valores de RMSE, AIC y BIC (Tabla 3), en el caso de las curvas de inactivación correspondientes a jugo de manzana. De los parámetros obtenidos se desprende nuevamente que el tratamiento por $\mathrm{LP}_{\mathrm{e}}$ fue más efectivo en el jugo de melón, dado que presentó mayores valores $\operatorname{de} f, \mathrm{k}_{\max 1} \mathrm{y}_{\max 2}$ (Tabla 1 ), que E. coli fue el microorganismo más sensible al tratamiento. Según este modelo, las curvas de supervivencia corres- 


\begin{tabular}{|c|c|c|c|c|c|}
\hline \multicolumn{2}{|c|}{ Tratamiento } & Moda (s) & Media (s) & Varianza (s $\left.{ }^{2}\right)$ & Coeficiente de Sesgo (-) \\
\hline \multirow{2}{*}{$\begin{array}{c}\text { Jugo de } \\
\text { manzana }\end{array}$} & E. coli & $(-)$ & 38,5 & +++ & 13,4 \\
\hline \multirow{4}{*}{ Jugo de melón } & S. cerevisiae & $(-)$ & 127,2 & +++ & 7,8 \\
& E. coli & $(-)$ & 2,3 & 33 & 7,1 \\
& S. cerevisiae & $(-)$ & 9,9 & 203 & 3,2 \\
& A. acidoterrestris & $(-)$ & 28,5 & +++ & 2,5 \\
\hline
\end{tabular}

Tabla 2. Estadísticos asociados al modelo de Weibulla, correspondientes a las curvas de inactivación de células de E. coli, $S$. cerevisiae y esporas de $A$. acidoterrestris en jugos frutales tratados $60 \mathrm{~s} \mathrm{LP}_{\mathrm{e}}$ a Estadísticos del modelo de Weibull, $t_{\mathrm{c}}$ media de la distribución, $\sigma_{t c}^{2}$ varianza, $v_{1}$ coeficiente de sesgo. ${ }^{b}+++$ Valor del estadístico mayor que 1000.

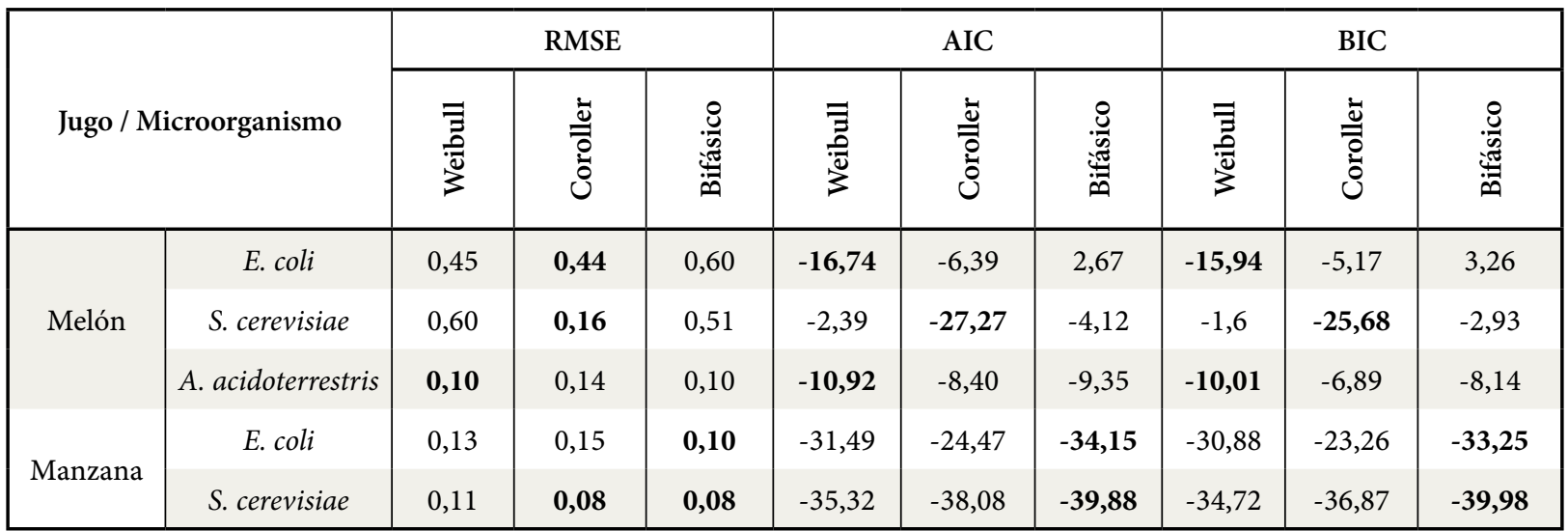

Tabla 3. Valores de los índices de ajuste RSME, AIC y BICa para las curvas de inactivación de los microorganismos ensayados en los jugos frutales tratados con $\mathrm{LP}_{\mathrm{e}}$.

pondientes a E. coli y S. cerevisiae en los jugos evaluados presentaron una tasa de inactivación muy pronunciada en los primeros $10 \mathrm{~s}$ de tratamiento, seguido por una segunda porción de la curva con menor tasa de inactivación que indica que el proceso se hace menos efectivo con el tiempo de procesamiento. En el caso de las A. acidoterrestris, se observa un primer período con una baja tasa de inactivación seguido de una segunda fase de mayor tasa de inactivación (Tabla 1), lo que indicaría en este caso que el proceso se vuelve más efectivo con el tiempo de exposición.

El modelo de Coroller fue el que representó mejor la curva sigmoidea correspondiente a $S$. cerevisiae inoculada en jugo en melón, con valores de AIC y BIC mucho menores respecto a los otros modelos. Es probable que en los restantes casos los criterios de AIC y BIC hayan penalizado el alto número de parámetros del modelo en el balance entre el ajuste y la parsimonia.

El análisis de los criterios de AIC y BIC determinaron que, en general, los modelos de Weibull y bifásico implicaron mejor ajuste, menor número de parámetros, o ambos.

La combinación US y $\mathrm{LP}_{\mathrm{c}}$ resultó aditiva en la inactivación de E. coli en jugo de manzana. En conformidad con estos resultados, Muñoz et al. (2012a) reportaron efecto aditivo en la inactivación de Listeria innocua en solución buffer ( $\mathrm{pH} 4,0)$ tratada por LP $(42,5 \mathrm{~J} / \mathrm{ml})$ combinada con termosonicación (TS, $500 \mathrm{~W}, 20 \mathrm{KHz}, 48-50^{\circ} \mathrm{C}, 100 \mathrm{~J} / \mathrm{ml}$ ) en sistema continuo. De la misma manera, Muñoz et al. (2011 y 2012b) estudiaron la combinación entre PL $\left(35^{\circ} \mathrm{C}\right.$, $\left.4,03-5,10 \mathrm{~J} / \mathrm{cm}^{2}\right)$ y TS $\left(24 \mathrm{KHz}, 400 \mathrm{~W}, 14 \mathrm{ml} / \mathrm{min}, 40{ }^{\circ} \mathrm{C}\right.$ o $8 \mathrm{ml} / \mathrm{min}, 53^{\circ} \mathrm{C}$ ) en la inactivación de $E$. coli en jugos de naranja y manzana en un sistema de flujo continuo y observaron que los tratamientos combinados presentaron efecto aditivo, alcanzando hasta $\sim 5,0$ y 3,2 reducciones log en jugos de manzana y naranja, respectivamente.

Es importante destacar que el tratamiento por $\mathrm{LP}_{c}$ fue más efectivo en la inactivación que $\mathrm{LP}_{\mathrm{e}}$, dado que se lograron 3,1 reducciones logarítmicas al utilizar la dosis máxima de $0,73 \mathrm{~J} / \mathrm{cm}^{2}$ de $\mathrm{LP}_{\mathrm{c}}$ en jugo de manzana, mientras que solo se obtuvo una reducción de 0,63 ciclos logarítmicos luego de la exposición a la dosis más baja de $\mathrm{LP}_{\mathrm{e}}$ analizada $\left(2,4 \mathrm{~J} / \mathrm{cm}^{2}\right)$ (Figuras 1 y 3 ).

El análisis de conglomerados permitió discriminar un grupo de consumidores interesados en el fuerte sabor a manzana y en la acidez del jugo de manzana procesado por el tratamiento combinado.

\section{Conclusiones}

Este estudio demostró que se obtuvieron importantes reducciones de algunos microorganismos de relevancia en jugos de manzana y melón en tiempos muy cortos (60 s) de tratamiento con LP en modalidad estanco. La inactivación mediante $\mathrm{LP}_{\mathrm{e}}$ dependió fuertemente del tipo de jugo empleado y del microorganismo, y el tratamiento fue más efectivo en el jugo de melón. Los modelos de Weibull y bifásico ajustaron ade- 
cuadamente las curvas de inactivación de $\mathrm{LP}_{\mathrm{e}}$, mientras que el modelo de Coroller presentó sobreparametrización en la mayoría de los casos, a excepción de las curvas de inactivación que resultaron sigmoideas. Los parámetros obtenidos explicaron, desde distintos puntos de vista, la influencia de esta tecnología en la inactivación microbiana.

La combinación de $\mathrm{LP}_{\mathrm{c}}$ con US en jugo de manzana resultó aditiva y alcanzó más de los 5 ciclos logarítmicos de reducción requeridos para $E$. coli. La segmentación de consumidores a través del análisis de conglomerados permitió discriminar un grupo de consumidores que presentó un fuerte interés en el jugo de manzana procesado por ultrasonido y luz pulsada en sistema continuo, quienes valoraron positivamente el intenso sabor a manzana y la acidez del producto.

\section{Referencias}

Anderson, J.G., Rowan, N.J., MacGregor, S.J., Fouracre, R.A., y Farish, O., 2000. Inactivation of food-borne enteropathogenic bacteria and spoilage fungi using pulsedlight. En: IEEE Transactions on Plasma Science, 28, pp.83-88.

Akaike, H., 1973. Proceedings of the 2nd International Symposium of Information. En: B.N. Petrov, y F. Cza'ki (Eds.). En: Information theory and extension of the maximum likelihood principle. Akademiai Kiado: Budapest, pp.267-281.

Alzamora, S.M., Guerrero, S., Viollaz, P., y Welti, J., 2005. Novel food processing. En: Barbosa-Cánovas G. (Ed.). Experimental protocols for modeling the response of microbial populations exposed to emerging technologies: Some points of concern. Nueva York, Marcel Dekker, Inc., pp.591-607.

Alzamora, S.M., Guerrero, S., Schenk, S., Raffellini, S. y LópezMalo, A., 2011. Inactivation of microorganisms. En: Feng H., Barbosa-Canovas G., Weiss J., (Eds.). Ultrasound Technologies for Food and Bioprocessing. Nueva York, Springer, pp.321-343.

Bahçeci, K., Gökmen, V., Acar, J., 2005. Formation of guaiacol from vanillin by Alicyclobacillus acidoterrestris in apple juice: a model study. En: European Food Research and Technology, 220, pp.196-199.

Cerf, O., 1977. Tailing of survival curves of bacterial spores. En: Journal of Applied Microbiology, 42, pp.1-19.

Coroller, L., Leguerinel, I., Mettler, E., Savy, N., y Mafart, P., 2006. General model, based on a two mixed Weibull distributions of bacterial resistance, for describing various shapes of inactivation curves. En: Applied and Environmental Microbiology, 72, pp.6493-6502.

Everitt, B.S., Landau, S., y Leese, M., 2001. Cluster analysis. , Oxford, John Wiley \& Sons.

Ferrante, S., Guerrero, S., y Alzamora, S.M., 2007. Combined use of ultrasound and natural antimicrobials to inactivate L. monocytogenes in orange juice. En: Journal of Food Protection, 70, pp.1850-1857.

Ferrario, M., Guerrero, S., y Alzamora, S.M. (2013a). Study of pulsed light-induced damage on Saccharomyces cerevisiae in apple juice by flow cytometry and transmission electron microscopy. En: Food and Bioprocess Technology, 7, pp.1001-1011.

Ferrario, M., Alzamora, S.M., y Guerrero, S., 2013b. Inactivation kinetics of some microorganisms in apple, melon, orange and strawberry juices by high intensity light pulses. En: Journal of Food Engineering, 118, pp.302-311.
Gómez, P., Salvatori, D., García Loredo, A., Alzamora, S.M., 2012. Pulsed light treatment of cut apple: dose effect on color, structure and microbiological stability, En: Food and Bioprocess Technology, 5, pp.2311-2322.

Gómez-López, V., Ragaert, P., Debevere, J., y Devlieghere, F., 2007. Pulsed light for food decontamination: a review. En: Trends in Food Science and Technology, 18, pp.464-473.

Guerrero, S., López-Malo, A., Alzamora, S.M., 2001. Effect of ultrasound on the survival of Saccharomyces cerevisiae: influence of temperature, $\mathrm{pH}$ and amplitude. En: Innovative Food Science and Emerging Technologies, 2, pp.31-39.

Guerrero, S., Tognon, M., Alzamora, S.M., 2005. Response of Saccharomyces cerevisiae to the combined action of ultrasound and low weight chitosan. En: Food Control, 16, pp.131-139.

Hierro, E., Barroso, E., De la Hoz, L., Ordóñez, J. Manzano, S. y Fernández, M., 2011. Efficacy of pulsed light for shelf-life extension and inactivation of Listeria monocytogenes on ready-to-eat cooked meat products. En: Innovative Food Science and Emerging Technologies, 3, pp.275-281.

Izquier, A., y Gómez-López, V., 2011. Modeling the pulsed light inactivation of microorganisms naturally occurring on vegetable substrates. En: Food Microbiology, 28, pp.1170-1174.

Jun, S., Irudayaraj, J., Demirci, A., y Geiser, D., 2003. Pulsed UV light treatment of corn meal for inactivation of Aspergillus niger spores. En: International Journal of Food Science and Technology, 38, pp.883-888.

Knorr, D., Zenker, M., Heinz, V., Lee, D., 2004. Applications and potential of ultrasonics in food processing. En: Trends in Food Science and Technology, 15, pp.261-266.

Krishnamurthy, K., Tewari, J., Irudayaraj, J., y Demirci, A., 2010. Microscopic and spectroscopic evaluation of inactivation of Staphylococcus aureus by pulsed light and infrared heating. En: Food and Bioprocess Technology, 3, pp.93-104.

Krishnamurthy, K., Demirci, A. y Irudayaraj, J., 2008. Inactivation of Staphylococcus aureus in milk using flowthrough pulsed UV-light treatment system. En: Food Microbiology and Safety, 72, pp.233-239.

Lasagabaster, A. y Martínez, I., 2014. Survival and growth of Listeria innocua treated by pulsed light technology: impact of posttreatment temperature and illumination. En: Food Microbiology, 41, pp.76-81.

Lawless, H., 2013. Segmentation. En: H. Lawless, (Ed.), Quantitative sensory analysis. Psychophysics, models and intelligent design. Oxford, Wiley Blackwell, pp.323-338.

Martínez, A., Díaz, R.V., y Tapia, M.S., 2000. Microbial ecology of spoilage and pathogenic flora associated to fruits and vegetables, En: Alzamora, S.M., Tapia, M., López-Malo, A. (Eds.), Minimally processed fruits and vegetables. Fundamental aspects and applications. Gaithersburg, Aspen publishers Inc., pp.43-62.

Muñoz, A., Palgan, I., Noci, F., Morgan, D., Cronin, D., Whyte, P. y Lyng, J., 2011. Combinations of high intensity light pulses and thermosonication for the inactivation of Escherichia coli in orange juice. En: Food Microbiology, 28, pp.1200-1204.

Muñoz, A., Palgan, I., Noci, F., Cronin, D.A., Morgan, D.J., Whyte, P. y Lyng, J., 2012a. Combinations of selected nonthermal technologies and antimicrobials for microbial inactivation in a buffer system. En: Food Research International, 47, pp.100-105. 
Muñoz, A., Caminiti, I., Palgan, I., Pataro, G., Noci, F., Morgan, D., Cronin, D., Whyte, P., Ferrari, G. y Lyng, J., 2012b. Effects on Escherichia coli inactivation and quality attributes in apple juice treated by combinations of pulsed light and thermosonication. En: Food Research International, 45, pp.299-305.

Pataro, G., Muñoz, A., Palgan, I., Noci, F., Ferrari, G. y Lyng, J.G., 2011. Bacterial inactivation in fruit juices using a continuous flow pulsed light (PL) system. En: Food Research International, 44, pp.1642-1648.

Peleg, M. y Cole, M. B., 1998. Reinterpretation of microbial survival curves. En: Critical Reviews in Food Science, 38, pp.353-380.

Piyasena, P., Mohareb, E. y McKellar, R., 2003. Inactivation of microbes using ultrasound. A review. En: International Journal of Food Microbiology, 87, pp.207-216.

Quinn, G. y Keough, M., 2002. Generalized linear models and logistic Regression. En: Quinn G. y Keough M., M. Experimental design and data analysis for biologists. Cambridge, Cambridge University Press, pp.359-380.

Sauer, A. y Moraru, C., 2009. Inactivation of Escherichia coli ATCC 25922 and Escherichia coli O157:H7 in apple juice and apple cider using pulsed light treatment. En: Journal of Food Protection, 72, pp.937-944.

Silva, F., Gibbs, P., 2001. Alyciclobacillus acidoterrestris spores in fruit products and design of pasteurization processes. En: Trends in Food Science and Technology, 12, pp.68-74.

Smit, Y., Cameron, M., Venter, P., Witthuhn, R., 2010. Alyciclobacillus spoilage and isolation- A review. En: Food Microbiology, 28, pp.331-349.

Stratford, M., Hofman, P., Cole, M., 2000. Fruit juices, fruit drinks, and soft drinks, En: Lund, B.M., Baired Parker, T.C., Gould, G.W. (Eds.). Microbiological safety and quality of food. Gaithersburg, Aspen Publishers Inc., pp.836-850.

Vercammen, A., Vivijs, B., Lurquin, I., Michiels, C., 2012. Germination and inactivation of Bacillus coagulans and Alicyclobacillus acidoterrestris spores by high hydrostatic pressure treatment in buffer and tomato sauce. En: International Journal of Food Microbiology, 152, pp.162-167.

Wekhof, A., 2000. Desinfection with flash lamps. En: PDA Journal of Pharmaceutical Science and Technology, 54, pp.264-275. 